\title{
Unravelling the Mechanistic Links between Pro-Arrhythmia and Mechanical Function
}

\author{
Hannah J Smith, Francesca Margara, Blanca Rodriguez \\ Department of Computer Science, University of Oxford, Oxford, UK
}

\begin{abstract}
Sudden cardiac death (SCD) from ventricular arrhythmias is a leading cause of mortality. Accurate arrhythmic risk stratification is vital for preventative clinical interventions. Ejection fraction $(E F)$ is the primary metric used, but its accuracy is under debate, as many $S C D$ cases exhibit preserved EF. Thus, identifying clear links between EF and arrhythmic risk is critical. Here, as a step forward, we investigate the ionic processes determining cellular pro-arrhythmic mechanisms and their relationship with active tension. A population of 2500 human ventricular electromechanical cellular models was created, and stimulated to produce pro-arrhythmic behaviour. We quantified their susceptibility to develop early afterdepolarizations (EADs) and action potential duration (APD) shortening, as key arrhythmic markers. The relationship between both arrhythmic markers and tension amplitude was found to be highly dependent on ionic mechanism. Variability in L-type calcium current was the primary determinant of active tension and arrhythmia susceptibility, alongside SERCA and hERG expression. Models with low tension could exhibit both high and low EAD susceptibility. APD shortening, however, displayed a weak positive correlation with active tension amplitude.
\end{abstract}

\section{Introduction}

Ventricular arrhythmias are a primary cause of unexpected, sudden cardiac deaths (SCDs), with at least half of events attributed to ventricular arrhythmias [1]. Implantable cardioverter-defibrillators and other preventative interventions can be used to mitigate risk of SCD, and reduce the 50-100 deaths they cause per 100,000 population in Europe and the USA [1]. However, these interventions carry non-trivial risk and cost, so only should be used for a patient with significantly increased risk of dangerous arrhythmias. Therefore, accurate risk stratification tools for arrhythmias are vital. One of the primary risk factors currently considered for SCD is a depressed ejection fraction (EF), but the relationship between EF and arrhythmic risk is not well understood while reduced $\mathrm{EF}$ is certainly associated with an increased risk of SCD, more than half of SCD victims have preserved $\mathrm{EF}[2]$.
The mechanisms regulating mechanical function and arrhythmic risk are complex, including ionic, cellular and tissue-level dynamics. As a first step, in this study, we aim to investigate the ionic mechanisms relating active tension (as a key determinant of EF) and pro-arrhythmic cellular markers including EADs, a risk factor for dangerous ventricular arrhythmias, including Torsade de pointes [3], and APD shortening, which is known to promote re-entrant arrhythmias [4]. To do so, we construct a population of cellular models based on a recently developed human cellular model that couples electrophysiology with contractile mechanisms. We hypothesise that variability in the L-type calcium current is a primary determinant of active tension, and also of pro-arrhythmic cellular properties such as EADs and APD shortening, with the former also strongly modulated by SR calcium reuptake and the latter by the hERG potassium current.

\section{Methods}

\subsection{Population of human electromechanical cell models}

As illustrated in Figure 1, two human-based models were coupled [5], namely, the new ToR-ORD human electrophysiology and excitation-contraction coupling endocardial cell model [6] and the Land-Niederer contractile model [7]. This allows the investigation of ionic mechanisms underlying both electrophysiology (such as cellular arrhythmic markers, and other changes to the AP and $\mathrm{CT}$ ) and tension biomarkers.

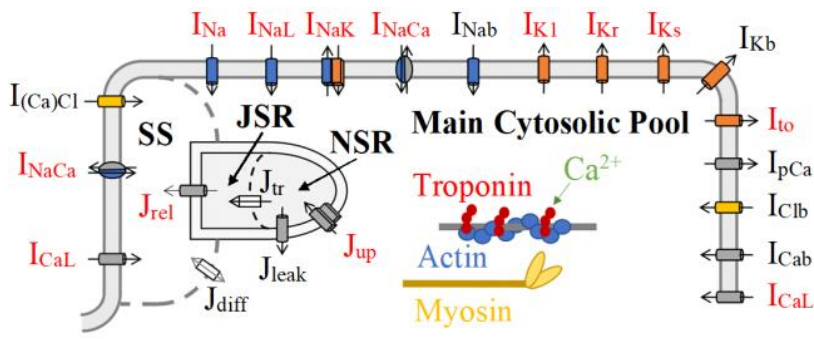

Figure 1. Schematic representation of the two humanbased coupled models: the ToR-ORD model with calcium dynamics and the Land-Niederer contractile model (adapted from [5]). Currents shown in red were varied. 
'SS' indicates junctional subspace compartment, where calcium influx via L-type calcium current occurs and where calcium is released from the sarcoplasmic reticulum. 'JSR' and 'NSR' are junctional and network sarcoplasmic reticulum compartments, respectively. 'Main cytosolic pool' is the remaining intracellular space.

A population of 2500 models was created by varying the following 11 ionic currents, as in [8]: $\mathrm{I}_{\mathrm{Na}}, \mathrm{I}_{\mathrm{NaL}}, \mathrm{I}_{\mathrm{Kr}}, \mathrm{I}_{\mathrm{Ks}}, \mathrm{I}_{\mathrm{K} 1}$, $\mathrm{I}_{\mathrm{NaCa}}, \mathrm{I}_{\mathrm{NaK}}, \mathrm{J}_{\mathrm{rel}}, \mathrm{J}_{\mathrm{up}}, \mathrm{I}_{\mathrm{CaL}}$ and $\mathrm{I}_{\mathrm{to}}$. Each current was modified by inserting a scaling prefactor, varied between the values of 0 and 2. This is analogous to changing the channel's conductance, though is primarily modelling the disparity in expression of channels on both an inter- and intrasubject basis. This range gives good variation in biomarkers over experimental ranges. The population was calibrated to enable diseased phenotypes, but with normal overall behaviour (at $1 \mathrm{~Hz}$ pacing with no drug block), such that its $\mathrm{APD}_{90}$ was within experimental ranges [3]. Thus, models with significant repolarization abnormalities were excluded by requiring that resting membrane potential did not exceed -60mV. Non-physiological calcium accumulation was prevented by discarding models whose calcium transient amplitude exceeded 2000nM.

\subsection{Simulation protocols}

Simulations were performed using the ode $15 \mathrm{~s}$ numerical integration function on Matlab due to its applicability for stiff ODEs. Each simulation was run for 200 beats, to allow the system to reach steady-state, and all biomarkers were calculated on the last beat. Four different pacing frequencies were used - basic cycle lengths (BCLs) of 300, 500,1000 and $4000 \mathrm{~ms}$, alongside separate hERG and Ltype calcium blocks of varying magnitude in order to stimulate pro-arrhythmic behaviour.
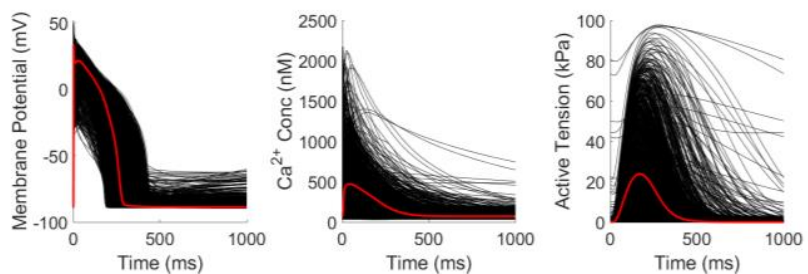

Figure 2. Simulated action potential (left), calcium transient (middle) and active tension (right) traces obtained from the calibrated population of electromechanical models (black traces) and the ToR-ORd/Land baseline model (red trace).

\subsection{Biomarkers}

Two cellular arrhythmic markers were tested - early afterdepolarizations (EADs) and reduced action potential duration (APD). EADs were classified where a second peak was found, after at least $150 \mathrm{~ms}$, whose prominence exceeds $10^{-4} \mathrm{mV}$ and peaks to $>0 \mathrm{mV}$, for a beat that repolarizes to <-60mV, as in [9]. An example of an EAD is shown in Figure 3. Models were scored on their susceptibility to develop EADs by the minimum degree of hERG block required to produce an EAD. A score of 0 was given if the model did not develop an EAD for any hERG block magnitude, 1 if the model only developed an EAD for the strongest block, up to a maximum score of 6 if the model developed an EAD even with no hERG block. Scores were assigned for both normal and slow pacing. $\mathrm{APD}_{90}$ was calculated, as low values are a risk factor for re-entry arrhythmias [3]. Tension amplitude $\left(\mathrm{T}_{\text {amp }}\right)$ was calculated as its resting value (the average over the last $50 \mathrm{~ms}$ of the AP) subtracted from its peak. Spearman's Rank correlation coefficient was calculated between biomarkers that had a monotonic relationship, due to the nonlinearity of these correlations.
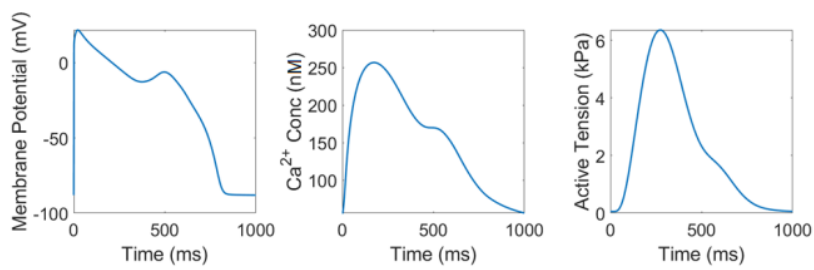

Figure 3. Cardiac model displaying an EAD. A significant second peak is present in the action potential (left), which is damped but remains present in the calcium transient (middle) and active tension (right).

\section{Results and Discussion}

\subsection{Key ionic drivers}

Figure 2 illustrates traces of the action potential, calcium transient and active tension for all models considered for the investigations. Their analysis revealed that L-type calcium current $\left(\mathrm{I}_{\mathrm{CaL}}\right)$, calcium reuptake via SERCA ( $\mathrm{J}_{\text {up }}$ and $\mathrm{I}_{\mathrm{Kr}}$ are the three ionic currents with strongest contributions to active tension amplitude, EAD susceptibility and APD shortening, in line with their clinical relevance. Results illustrating their relationships are shown in Figure 4.

L-type calcium current $\left(\mathrm{I}_{\mathrm{CaL}}\right)$ was identified as one of the primary drivers of the relationship between active tension and arrhythmic risk. As shown in Figure 4b, increasing $\mathrm{I}_{\mathrm{CaL}}$ results in a significant increase in active tension. This is in line with the primary role of $\mathrm{I}_{\mathrm{CaL}}$ in calcium influx and systolic intracellular calcium concentration. This increased expression occurs during beta adrenergic stimulation [10] and gives a marked increase in EAD susceptibility (Figure 4a). Reduced expression is associated with moderate APD shortening (Figure 4a).

Uptake of calcium into the SR via SERCA $\left(J_{\text {up }}\right)$ also has a significant impact on the active tension amplitude, with reduced expression (as seen in heart failure [11]) associated with a significant decrease in tension (Figure 
4d). This is primarily due to two effects - the diastolic intracellular calcium concentration increases due to reduced reuptake while the systolic calcium concentration significantly falls due to reduced SR release. Both high and low expressions of SERCA lead to increases in EAD susceptibility, though it has little impact on the APD (Figure 4c).

The rapid delayed inwardly rectifying potassium current ( $\mathrm{I}_{\mathrm{Kr}}$ ) via $\mathrm{hERG}$ is particularly critical for determining APD. Significant APD shortening is observed for increased $\mathrm{I}_{\mathrm{Kr}}$ expression (Figure 4e). This is not associated with a decrease in tension amplitude, with significant tension decreases only observed in models with very low hERG expressions (Figure 4f).

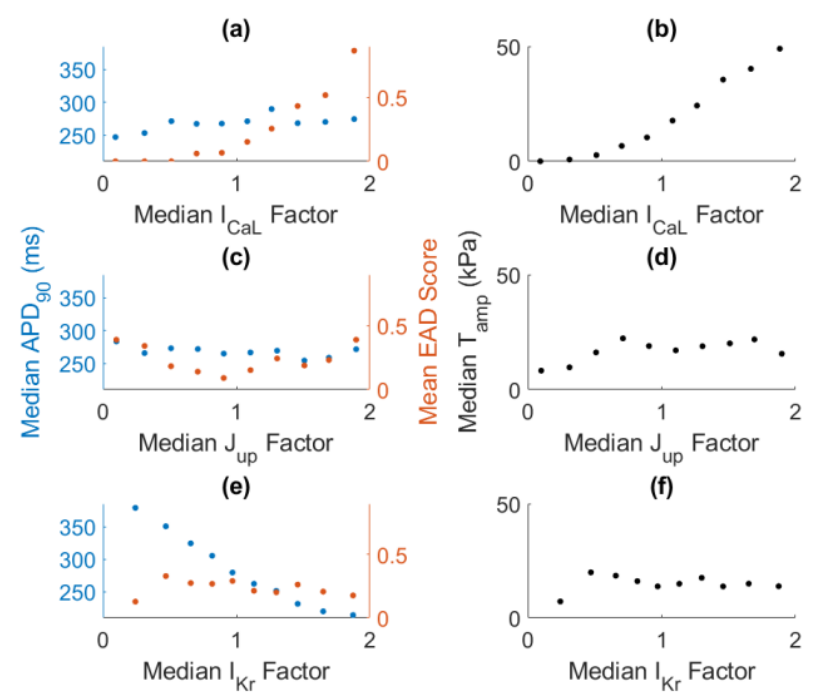

Figure 4. Variation of $\mathrm{APD}_{90}, \mathrm{EAD}$ susceptibility and active tension amplitude with each key current magnitude. Population was split into deciles for the three ionic scaling factors shown and the average biomarkers were calculated for each decile. Upper: L-type calcium current, middle: calcium reuptake via SERCA, lower: rapid delayed inwardly rectifying potassium current via $h E R G$. $T_{a m p}$ increases with $\mathrm{I}_{\mathrm{CaL}}(\rho=0.77)$ and $\mathrm{APD}_{90}$ decreases with $\mathrm{I}_{\mathrm{Kr}}$ $(\rho=-0.67)$.

\subsection{EADs and active tension}

Low tension values are largely observed for models with low EAD susceptibility (i.e. low scores), as shown in Figure 5. Thus, models can display a significant EAD susceptibility with normal, or even elevated, tension amplitude. As expected, EADs were easier to induce with slow pacing (i.e. higher scores for $\mathrm{BCL}=4000 \mathrm{~ms}$ ). The low tension amplitude seen for models displaying reduced EAD susceptibility are largely influenced by L-type calcium current. This is supported by the fact that models with lowest EAD susceptibility had both reduced $\mathrm{I}_{\mathrm{CaL}}$ expression (median $\mathrm{I}_{\mathrm{CaL}}$ scaling factor $=0.85$, compared with 0.99 for the population) and reduced tension amplitude $\left(\mathrm{T}_{\mathrm{amp}}=11.7 \mathrm{kPa}\right.$, population $\left.=14.6 \mathrm{kPa}\right)$ under normal pacing. These findings are even clearer for models that have the lowest EAD susceptibility under slow pacing, as median $\mathrm{I}_{\mathrm{CaL}}$ scaling factor was reduced further to 0.65 and $\mathrm{T}_{\text {amp }}$ to $6.8 \mathrm{kPa}$. Tension is highest for models with moderately increased EAD susceptibility (score 5 in Figures $5 \mathrm{a}$ and $5 \mathrm{~b}$ ), so preserved active tension certainly does not exclude increased EAD risk.

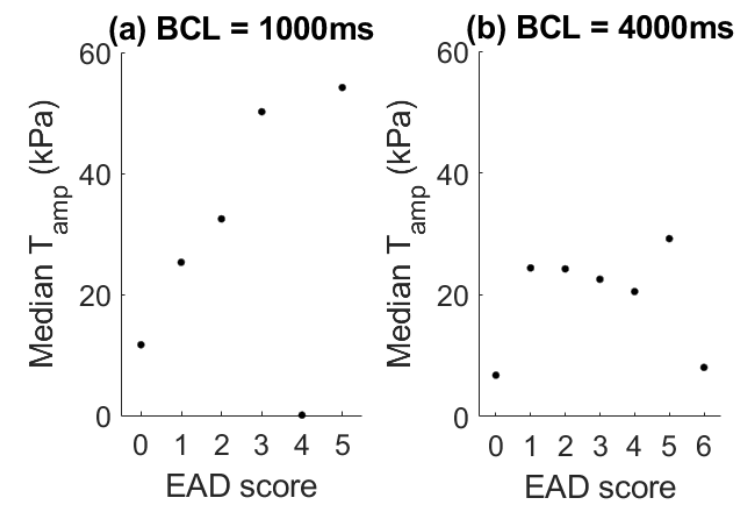

Figure 5. Median active tension amplitude versus EAD susceptibility score for both normal (a) and slow pacing (b). Decreased tension is seen with both high and low EAD susceptibility. No models in the calibrated population were given an EAD score above 5 under normal pacing.

\subsection{APD Shortening and active tension}

$\mathrm{APD}_{90}$ has an overall positive correlation with tension, as shown in Figures 6a and 6b. This means that while APD shortening is somewhat associated with decreases in active tension, again preserved active tension does not exclude increased arrhythmic risk. As with EAD susceptibility, this seems to reflect the trends seen in the L-type calcium current, where low expression leads to both low active tension and APD shortening.

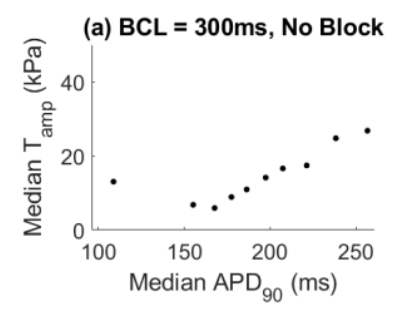

(b) $\mathrm{BCL}=1000 \mathrm{~ms}$, No Block
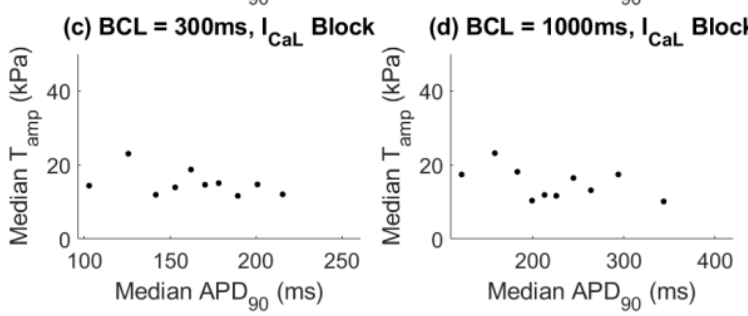

Figure 6. Median active tension amplitude versus $\mathrm{APD}_{90}$ shortening score for both fast (left) and normal pacing 
(right). Population was split into deciles based on $\mathrm{APD}_{90}$ and median active tension amplitude was calculated for each decile. Upper: no L-type calcium block, $\mathrm{T}_{\mathrm{amp}}$ increases slightly with $\operatorname{APD}_{90}(\rho=0.20$ for both fast and normal pacing), lower: strong L-type calcium block applied and tension decrease with APD shortening is lost.

This is supported by the fact that when a strong L-type calcium current block is used, the relationship between APD shortening and low tension is lost, as shown in the Figures $6 \mathrm{c}$ and $6 \mathrm{~d}$.

\section{Conclusion}

Simulations show that low tension values can be associated with APD shortening, which is a risk factor for developing re-entry arrhythmias, but they are not well correlated with increased EAD risk. The link between single cellular arrhythmic markers and active tension amplitude is highly dependent on its ionic mechanism - for example the same pro-arrhythmic behavior (increased EAD susceptibility) was found to be associated with high tension when caused by high L-type calcium current, but low tension with reduced SERCA expression. L-type calcium current plays a critical role in linking arrhythmic risk with active tension, which was demonstrated by the elimination of the correlation between APD shortening and active tension when an $\mathrm{I}_{\mathrm{CaL}}$ block was applied. Preserved active tension absolutely does not exclude increased arrhythmic risk, as increased tension amplitude was observed with moderately high EAD risk.

Thus, while active tension effects on EF would seemingly have a place for stratifying arrhythmic risk of ionic origin, caution is needed, as some pro-arrhythmic profiles actually cause high active tension. Further work is needed to investigate how the combination of specific ionic profiles with pro-arrhythmic structural changes would affect the EF. While structural causes of ventricular arrhythmias tend to come with a higher mortality than conditions of purely electrophysiological origin [2], electromechanical feedback means that these conditions often also present with pathology in their ionic expressions. The resulting EF observed is likely to be affected by both structural and ionic factors, which may have opposing effects, as some ionic changes increase the single cellular active tension amplitude. This interplay may contribute to the disparity in EF seen in patients with the same disease condition, and the failure of depressed EF to accurately identify all patients with high arrhythmic risk. Future investigations should simultaneously model both mechanical and electrophysiological abnormalities to investigate this combined effect of mechanical and ionic causes of arrhythmias.

\section{Acknowledgements}

HS is supported by a Wellcome Trust grant. FM is funded by the Personalised In-Silico Cardiology (PIC) project, European Union's Horizon 2020 research and innovation programme under the Marie SklodowskaCurie grant agreement 764738. BR is supported by a Wellcome Trust Fellowship in Basic Biomedical Sciences.

\section{References}

[1] P. Harris, D. Lysitsas, "Ventricular arrhythmias and sudden cardiac death," BJA Education, vol. 16, no. 7, pp. 221-229, July 2016.

[2] G. Lopera, A. B. Curtis, "Risk stratification for sudden cardiac death: Current approaches and predictive value," Curr. Cardiol. Rev., vol. 5, no. 1, pp. 56-64, Jan. 2009.

[3] E. Passini et al., "Human in silico drug trials demonstrate higher accuracy than animal models in predicting clinical pro-arrhythmic cardiotoxicity," Front. Physiol., vol. 8, pp. 668, Sept. 2017.

[4] U. Ravens, E. Cerbai, "Role of potassium currents in cardiac arrhythmias," EP Europace, vol. 10, no. 10, pp. 1133-1137, Oct. 2008.

[5] F. Margara et al., "In-silico human electro-mechanical ventricular modelling and simulation for drug-induced proarrhythmia and inotropic risk assessment," Prog. Biophys. Mol. Biol., issn. 0079-6107, July 2020.

[6] J. Tomek et al., "Development, calibration, and validation of a novel human ventricular myocyte model in health, disease, and drug block," eLife, art. no. e48890, Dec. 2019.

[7] S. Land et al., "A model of cardiac contraction based on novel measurements of tension development in human cardiomyocytes," J. Mol. Cell. Cardiol., vol. 106, pp. 68-83, May 2017.

[8] O. J. Britton et al., "Quantitative comparison of effects of dofetilide, sotalol, quinidine, and verapamil between human ex vivo trabeculae and in silico ventricular models incorporating inter-individual action potential variability," Front. Physiol., vol. 8, pp. 597, Aug. 2017.

[9] D. J. Schulz et al., "Variable channel expression in identified single and electrically coupled neurons in different animals," Nat. Neurosci., vol. 9, no. 3, pp. 356-362, Jan. 2006.

[10] M. A. G. van der Heyden et al., "Molecular aspects of adrenergic modulation of cardiac L-type $\mathrm{Ca}^{2+}$ channels," Cardiovascular Research, vol. 65, no. 1, pp. 28-39, Jan. 2005.

[11] A. R. Marks, "Calcium cycling proteins and heart failure: mechanisms and therapeutics," J. Clin. Invest., vol. 123, no. 1, pp. 46-52, Jan. 2013.

Address for correspondence:

Hannah J Smith.

Department of Computer Science, University of Oxford, Wolfson Building.

hannah.smith@msdtc.ox.ac.uk 